\title{
Mid-infrared dual-comb spectroscopy with GHz resolution using soliton microcombs
}

\author{
Chengying Bao, ${ }^{1, \dagger}$ Zhiquan Yuan, ${ }^{1, \dagger}$ Lue Wu ${ }^{1}$, Myoung-Gyun Suh ${ }^{1}$, Qiang Lin $^{2}$, and \\ Kerry Vahala ${ }^{1, *}$ \\ ${ }^{1}$ T. J. Watson Laboratory of Applied Physics, California Institute of Technology, Pasadena, CA 91125, USA \\ ${ }^{2}$ Department of Electrical and Computer Engineering, University of Rochester, Rochester, NY 14627, USA \\ ${ }^{\dagger}$ These authors contributed equally to this work. \\ *vahala@caltech.edu
}

\begin{abstract}
Microcomb based dual-comb spectroscopy of methane in the mid-infrared is demonstrated with $\mathrm{GHz}$ resolution. This fine resolution is enabled by generating spectrally densified mid-infrared combs via interleaved difference-frequency-generation. (C) 2021 The Author(s)
\end{abstract}

Dual-comb spectroscopy (DCS) is a powerful technique for precision measurement of gas spectra [1]. And soliton microcombs have recently been used to implement this method [2]. Ultimately, chip-based DCS modules using microcombs could revolutionize field measurement of various gases including chemical threats and greenhouse gases such as $\mathrm{CO}_{2}$ and $\mathrm{CH}_{4}$. In the application of DCS to gases, it is favorable to operate in the mid-infrared (mid-IR) where the gases have much stronger absorption [3]. And mid-IR silicon microcomb DCS has been reported with a line spacing of $127 \mathrm{GHz}$ [4]. Such a large line spacing is a result of the small microresonator form factor and causes spectral under-sampling of gas absorption lines whose bandwidths are usually narrower than tens GHz. Attaining direct generation of narrow line spacing microcombs in the mid-IR has so far not been possible. Interleaved difference-frequency-generation (iDFG) is a recently reported alternative approach for generation of mid IR comb spectra [5]. By selecting two near-infrared (near-IR) combs having repetition rates $f_{r}$ and $(N-$ 1) $f_{r} / N$, a temporal interleaving effect upon difference frequency generation is created, and the resulting mid-IR comb is densified to a line spacing of $f_{r} / N$.

Here, we use iDFG densified microcombs for mid-IR DCS with GHz resolution. A pair of counter-propagating (CP) solitons with repetition rates of $f_{r}^{\mathrm{cw}}$ and $f_{r}^{\mathrm{ccw}}$ were generated in a $22 \mathrm{GHz}$ silica wedge microresonator [6]. The locking dynamics between CP solitons that stabilize $\Delta f_{r}=f_{r}^{\mathrm{cw}}-f_{r}^{\mathrm{ccw}}$ [6] enhance the coherence of the resulting DCS system. The two soliton streams were then photodetected to drive a pair of electro-optical combs (EO-combs) at the rates of $(N-1) f_{r}^{\mathrm{cw}} / N$ and $(N-1) f_{r}^{\mathrm{ccw}} / N(N=8$ or 16) for iDFG (see experimental setup in Fig. 1(a)). The two near-IR combs operated at $1.55 \mu \mathrm{m}$ (soliton microcomb) and $1.06 \mu \mathrm{m}$ (EO-comb), thus generating mid-IR combs in the $3.3 \mu \mathrm{m}$ upon iDFG.
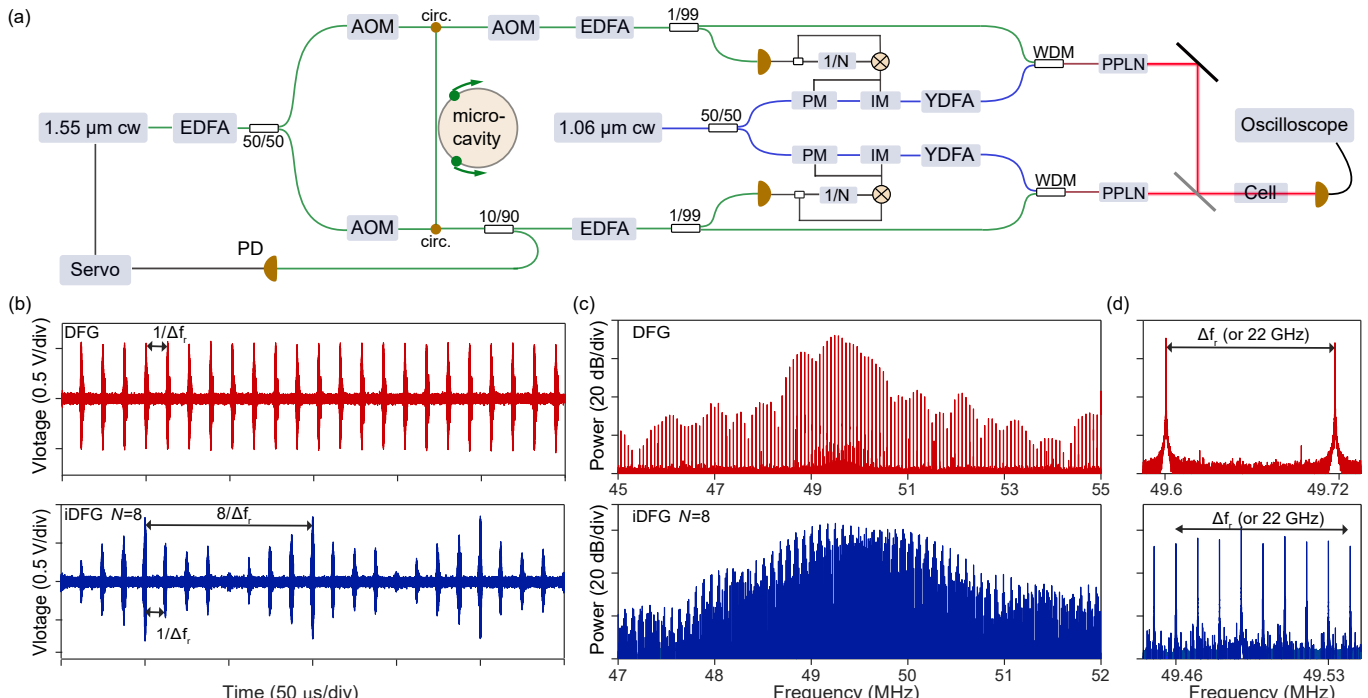

Fig. 1. (a) Experimental layout for iDFG DCS. PM (IM): phase (intensity) modulator, AOM: acousto-optical modulator, WDM: wavelength division multiplexer, circ:. circulator. (b) The midIR interferogram of conventional DFG and iDFG $(N=8)$. (c) The corresponding RF spectra. (d) Zoom in of the spectra in panel c for a RF bandwidth of about $\Delta f_{r}$ or $22 \mathrm{GHz}$ optical bandwidth. 

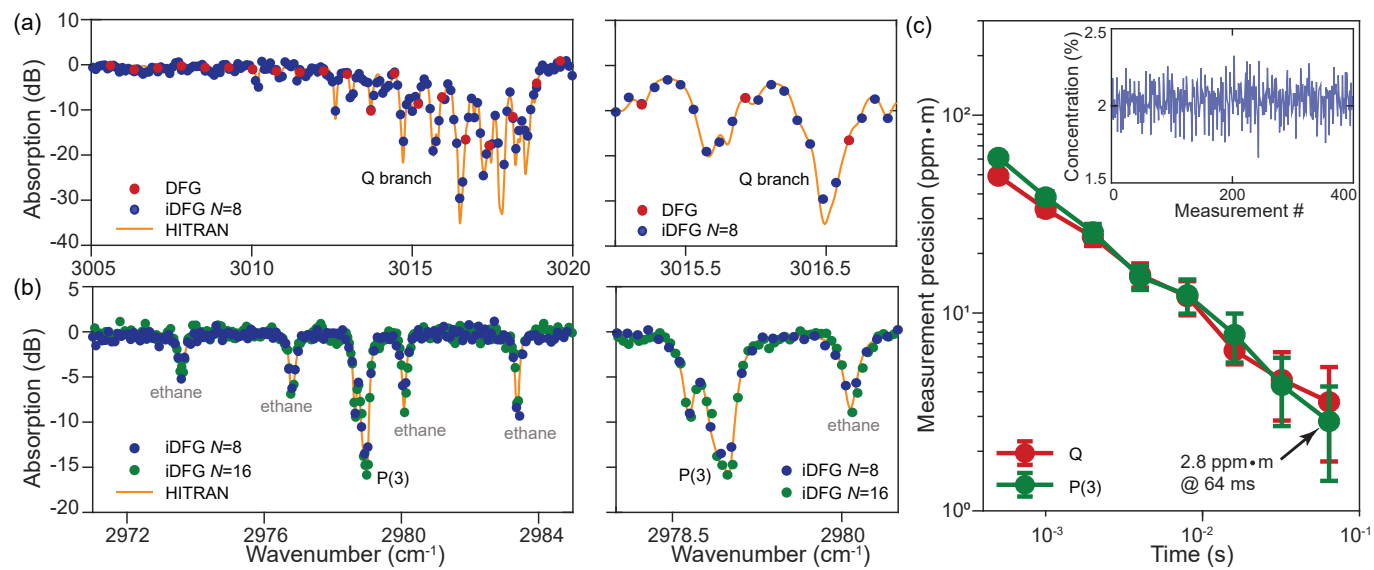

Fig. 2. (a) DCS of methane Q branch using conventional DFG and iDFG $N=8$. Hitran spectra are also provided. (b) DCS of methane $\mathrm{P}(3)$ and ethane using iDFG with $N=8$ and $N=16$. The right panels of $(a, b)$ are zoom-in of the spectra. (c) Measurement precision of the methane concentration for $\mathrm{iDFG} N=8$. The inset shows the sequence of methane concentration measured within $0.5 \mathrm{~ms}$ for $\mathrm{P}(3)$ branch using iDFG $N=8$.

The $1.06 \mu \mathrm{m}$ EO-comb was seeded by a tunable external cavity diode laser and its center wavelength was fine adjusted to probe different methane absorption branches. The soliton microcomb and the EO-comb were combined to pump a PPLN crystal for iDFG. The generated mid-IR combs were combined in free space to pass through a $5 \mathrm{~cm}$ long gas cell with a mixture of $2 \%$ methane and $0.5 \%$ ethane (buffered by nitrogen to a pressure of 760 torr). Their detected interferogram (without cell) was recorded by an oscilloscope. The interferograms for conventional DFG (continuous wave input at $1.06 \mu \mathrm{m}$ with EO-combs turned off) and iDFG are shown in Fig. 1(b). The interferogram repeats at the rate of $\Delta f_{r}$ (about $80 \mathrm{kHz}$ in experiment) for conventional DFG and at a rate of $\Delta f_{r} / N$ when iDFG is used. Transforming the interferograms into the frequency domain yields the RF spectra shown in Fig. 1(c). Spectral zoom-in illustrates the densification of the line spacing (Fig. 1(d)). The measured RF spectra also exhibit high SNR for iDFG with the peak SNR reaching 6800/ $\sqrt{s}$.

The gas absorption spectra were measured by normalizing the dual-comb spectra obtained with the gas cell by the spectra obtained without the gas cell. The measurement time is $200 \mathrm{~ms}$. We first measured the Q branch of methane around $3015 \mathrm{~cm}^{-1}$. The DCS results using DFG and iDFG $(N=8)$ are shown in Fig. 2(a). The iDFG case enables measurement of the fine structure within the Q branch with a resolution of about $2.8 \mathrm{GHz}$, while the conventional DFG case strongly undersamples the absorption features. Similarly, the mixed absorption spectra of methane and ethane around $2980 \mathrm{~cm}^{-1}$ were also measured using iDFG DCS (Fig. 2(b)). The system can be reconfigured for different resolutions by selecting different $N$ in the iDFG process. For example, using $N=16$ to produce a $1.4 \mathrm{GHz}$ mid-IR comb enables better resolution of the ethane absorption, which has a narrower linewidth than methane (see zoom-in of Fig. 2(b)). The measurement precision was evaluated by analyzing the Allan deviation of the measured methane concentration in the $5 \mathrm{~cm}$ cell (concentration measured in $0.5 \mathrm{~ms}$ used as a single data point, see inset of Fig. 2(c)). The analysis shows that a measurement precision of $2.8 \mathrm{ppm} \cdot \mathrm{m}$ was reached in $64 \mathrm{~ms}$ averaging time.

In summary, we demonstrated mid-IR DCS of methane with GHz resolution using the iDFG method. CP soliton microcombs in combination with EO-combs were employed. The system still uses a considerable amount of fiber optic components. However, a fully integrated device is potentially feasible. For example, the soliton microcomb, EO-modulators and PPLN waveguides have all been demonstrated using thin-film $\mathrm{LiNbO}_{3}$ devices.

This work was supported by the DTRA (HD-TRA11810047), the AFSOR (FA9550-18-1-0353), the Resnick Institute at Caltech, and the Kavli Nanoscience Institute.

\section{References}

1. I. Coddington, N. Newbury and W. Swann, Optica 3, 414 (2016).

2. M-G. Suh, Q-F Yang, K. Y. Yang, X. Yi, and K. Vahala, Science, 354, 600-603 (2016).

3. G. Ycas, et al., Nat. Photon. 12, 202 (2018).

4. M. Yu, Y. Okawachi, A. Griffith, N. Picqué, , M. Lipson, and A. Gaeta, Nat. Commun., 9, 1869 (2018).

5. C. Bao, Z. Yuan, H. Wang, L. Wu, B. Shen, K. Sung, S. Leifer and K. Vahala, Optica, 6, 309 (2020).

6. Q. F. Yang, X. Yi, K. Y. Yang, and K. Vahala, Nat. Photon., 11, 560 (2017). 